\title{
Excited-state dynamics and nonlinear optical response of Ge nanocrystals embedded in silica matrix
}

\author{
Luca Razzari, Andrea Gnoli, and Marcofabio Righini ${ }^{\mathrm{a})}$ \\ Istituto dei Sistemi Complessi-CNR Sezione di Roma-Tor Vergata, via del Fosso del Cavaliere 100, 00133 \\ Roma, Italy \\ Aykutlu Dâna and Atilla Aydinli \\ Physics Department, Bilkent University, Bilkent, 06800 Ankara, Turkey
}

(Received 15 February 2006; accepted 20 March 2006; published online 1 May 2006)

\begin{abstract}
We use a dedicated $Z$-scan setup, arranged to account for cumulative effects, to study the nonlinear optical response of Ge nanocrystals embedded in silica matrix. Samples are prepared with plasma-enchanced chemical-vapor deposition and post-thermal annealing. We measure a third-order nonlinear refraction coefficient of $\gamma=1 \times 10^{-16} \mathrm{~m}^{2} / \mathrm{W}$. The nonlinear absorption shows an intensity-independent coefficient of $\beta=4 \times 10^{-10} \mathrm{~m} / \mathrm{W}$ related to fast processes. In addition, we measure a second $\beta$ component around $10^{-9} \mathrm{~m} / \mathrm{W}$ with a relaxation time of $300 \mu \mathrm{s}$ that rises linearly with the laser intensity. We associate its origin to the absorption of excited carriers from a surface-defect state with a long depopulation time. (C) 2006 American Institute of Physics.
\end{abstract} [DOI: $10.1063 / 1.2201550]$

In recent years, linear and nonlinear optical properties of semiconductor nanocrystals (NCs) have attracted scientific attention driven both by fundamental and technological interests. To date, several preparation methods have been implemented to realize semiconductor NCs with narrow and reproducible size distribution. ${ }^{1}$ Altering the average NC size allows a broad modification of their energy structure and in particular, the energy band gap. Moreover, three-dimensional quantum confinement results in discrete energy structures and atomiclike behavior for NC optical transitions. Strong and fast optical nonlinearities, and strong photo- and electroluminescence, have been observed on materials composed of semiconductor NCs. ${ }^{2}$

In this context, group IV semiconductors like $\mathrm{Si}$ and Ge are principally studied with the aim to increase the radiation efficiency of indirect optical transitions by reducing the semiconductor size down to a nanometer scale. The potential applications of these materials in optoelectronics and microelectronics offer the main advantage of being compatible with conventional integrated circuit technology. Moreover, the exciton Bohr radius of bulk Ge $(24.3 \mathrm{~nm})^{3}$ is much larger than that of bulk $\mathrm{Si}(4.9 \mathrm{~nm}){ }^{4}$ This condition makes it easier to tailor electronic structure and optical properties of Ge-NC based materials by means of quantum size effects. The GeNCs embedded in silica matrices have shown visible photoluminescence $(\mathrm{PL})$ attributed to the quantum confinement mechanism. ${ }^{5}$ Furthermore, measurements of large third-order optical nonlinearities of the $\mathrm{Ge}-\mathrm{NC}$ have been recently reported. ${ }^{6-10}$ However, the understanding of the nonlinear optical response (NLO) in these materials is still not well established.

In this letter, we report on the study of the NLO of the Ge nanocrystals embedded in silica matrix. Samples were prepared by growing a germanosilicate film (Ge atomic fraction: 13.2\%) of $460 \mathrm{~nm}$ on a quartz substrate by PECVD. Samples were then annealed at temperatures ranging from

${ }^{a)}$ Electronic mail: marcofabio.righini@isc.cnr.it
650 to $850{ }^{\circ} \mathrm{C}$ for $5 \mathrm{~min}$. The NLO investigation has been performed using a dedicated setup of the $Z$-scan technique. In contrast with the standard $Z$-scan configuration proposed by Sheik-Bahae, ${ }^{11}$ closed-aperture (CA) and open-aperture (OA) curves are acquired by means of a digital oscilloscope. As described in detail in Ref. 12, the possibility to record the evolution in time of the $Z$-scan signals allows us to measure the dynamics of physical effects that persist in time more than the delay between successive laser pulses and hence accumulate. Furthermore, we can uncouple and separately measure single-pulse and cumulative effects. In the experiments reported here, the laser source was a mode-locked Ti:sapphire laser (Coherent MIRA 900-F). Laser pulses were $120 \mathrm{fs}$ wide, the repetition rate was $76 \mathrm{MHz}$, and the $\mathrm{cw}$ power was about $1 \mathrm{~W}$. The laser was modulated by a mechanical chopper. All measurements were performed at a wavelength of $800 \mathrm{~nm}$.

It is common knowledge that in $Z$-scan measurements with high repetition rate lasers, the real part of the refractive index could be altered by cumulative sample heating, giving rise to the time evolution of the CA curve. On the other hand, the OA curve usually does not evolve in time regardless of the laser repetition rate since optical absorption is generally weakly influenced by a change in the sample temperature. However, we observe clear time evolution on both CA and OA curves of samples containing Ge-NC. Neither time evolution or nonlinearity are measurable on a quartz reference sample. In Fig. 1, we report some $Z$-scan curves taken with different delays with respect to the beginning of the laser pulse train $(t=0$, corresponding to the opening of the chopper blade). For each $z$ position of the sample, a $1 \mathrm{~ms}$ trace of the intensity collected by the CA and OA detectors is acquired (see the insets of Fig. 1). An exponential fit of these traces allows us to extrapolate the curves at $t=0$ and at $t=\infty$. Curves obtained in this way are representative of single-pulse and cumulative effects, respectively, and are used to estimate the values of $\gamma$ (nonlinear refractive index) and $\beta$ (nonlinear absorption coefficient) at the corresponding times. ${ }^{12}$ 


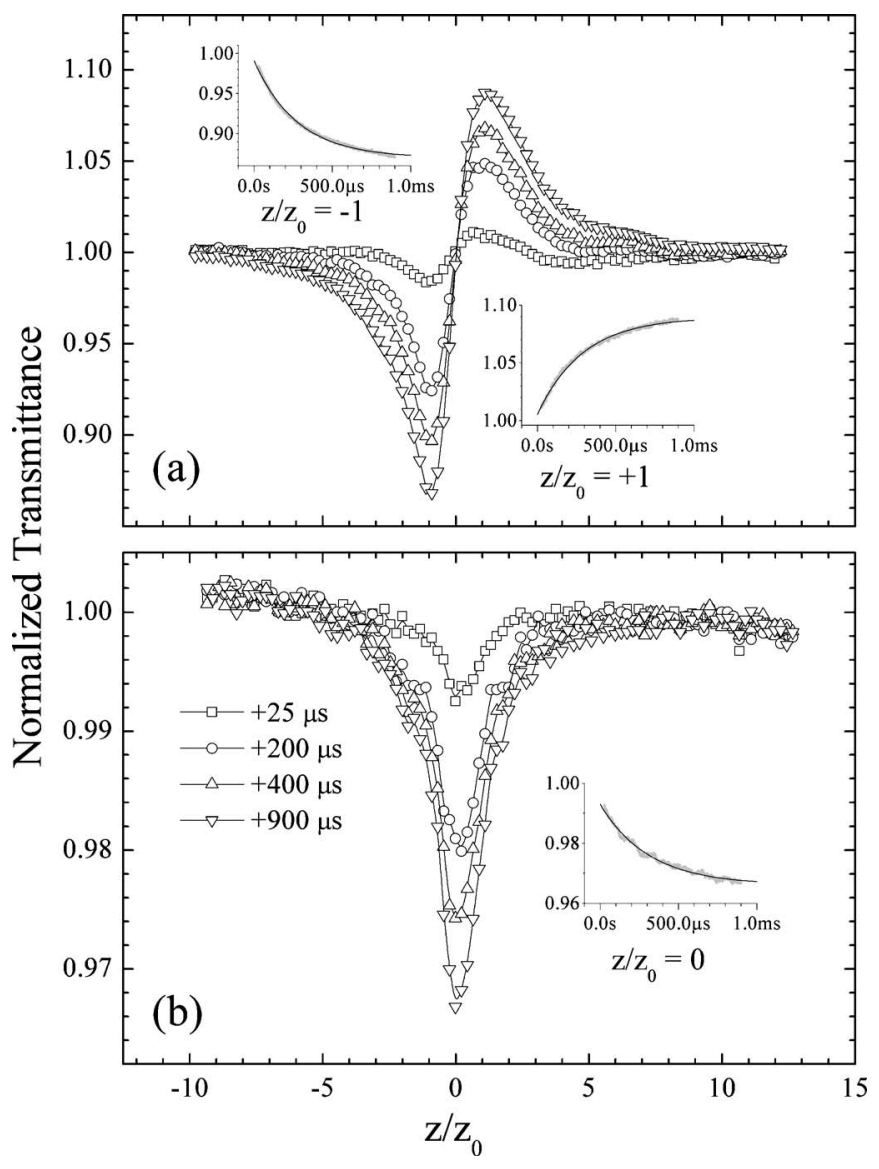

FIG. 1. Typical (a) closed- and (b) open-aperture Z-scan curves at different delays with respect to the chopper opening. In the insets, time evolutions at the most significant $z$ positions are also shown.

The time constants extracted from fitting CA and OA traces at each $z$ position for a sample annealed at $T$ $=850{ }^{\circ} \mathrm{C}$ are shown in Fig. 2. In general, the characteristic time constant $t_{c}$ of a thermal process due to laser heating in $Z$-scan is

$$
t_{c}=\frac{w^{2}(z)}{4 D}=\frac{w_{0}^{2}}{4 D}\left[1+\left(\frac{\lambda z}{\pi w_{0}^{2}}\right)^{2}\right],
$$

where $w_{0}$ is the beam waist, $w(z)$ is the beam radius at a specific $z$ position, $\lambda$ is the wavelength, and $D$ is the thermal

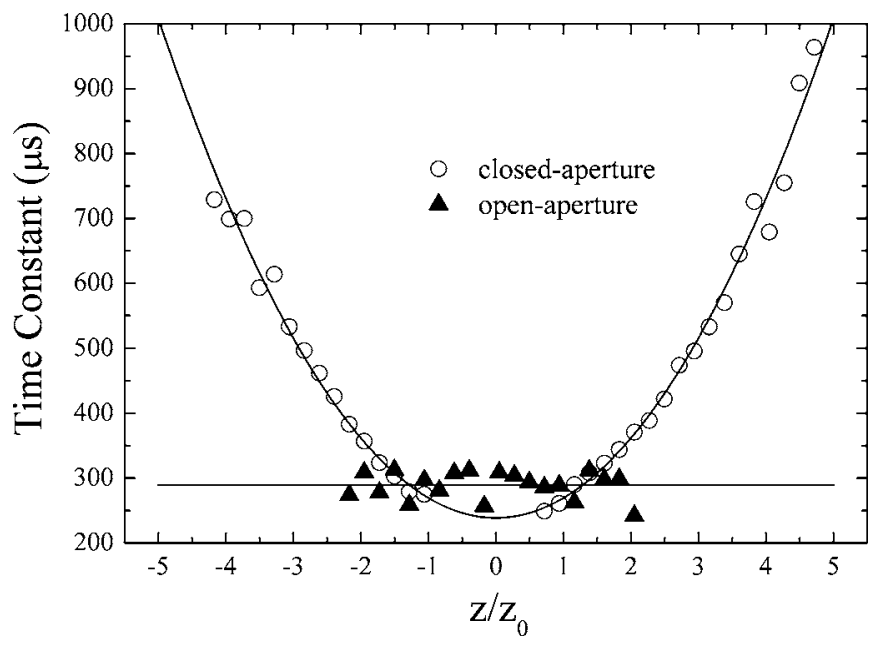

FIG. 2. Characteristic time constant of both closed- and open-apertures as a function of the normalized $z$ position for a sample annealed at $T=850{ }^{\circ} \mathrm{C}$.

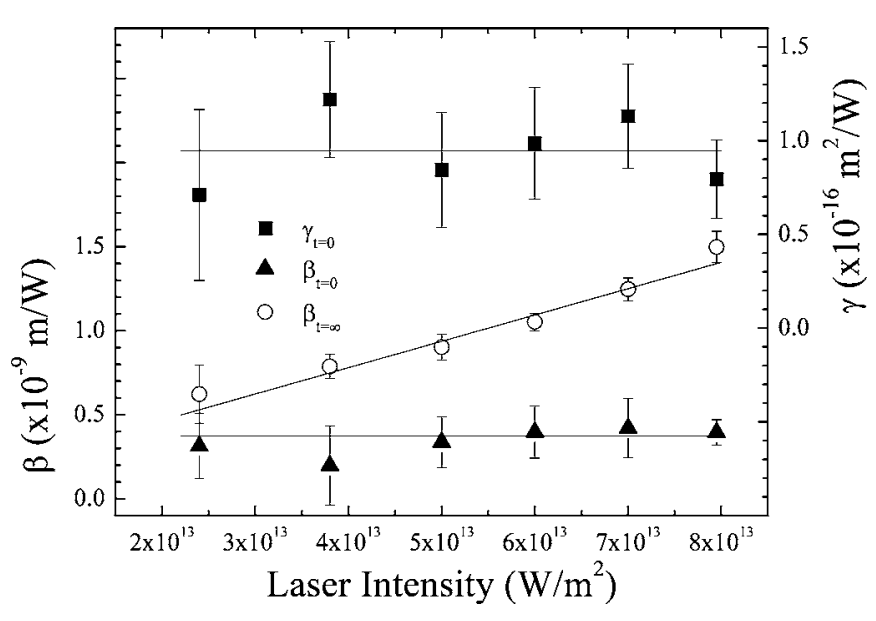

FIG. 3. Intensity dependence of single-pulse and cumulative nonlinear coefficients for a sample annealed at $T=850{ }^{\circ} \mathrm{C}$.

diffusion coefficient of the material being tested. As expected, the CA time constants follow the parabolic behavior predicted by Eq. (1) quite well. As a consequence, $\gamma_{t=\infty}$ is ascribable to the standard thermal-lensing effect. From the data of Fig. 2, we measure a value of $D=1.9 \times 10^{-7} \mathrm{~m}^{2} / \mathrm{s}$. The OA time constant, instead, does not show any change at different $z$ positions, being approximately $300 \mu$ s in the entire range. This confirms that the observed variation with time of the nonlinear absorption process is not related to a thermal effect. It is likely that the nonlinearity $\beta_{t=\infty}$ (i.e., the cumulative nonlinear absorption coefficient) is associated with the absorption of excited carriers from a trap state, the measured time constant being the state population lifetime.

Previous NLO studies carried out at $800 \mathrm{~nm}$ on similar Ge-NC systems observed population dynamics on faster time scales. A time-resolved degenerate four-wave mixing (DFWM) experiment has shown two distinct relaxation times: ${ }^{6}$ a fast one, on a time scale $<100 \mathrm{fs}$, ascribed to fieldinduced polarization and a slower response of about 1 ps. More recently, an experiment performed with $Z$-scan and pump and probe techniques ${ }^{7}$ confirmed a decay time of about 1 ps. The authors attribute this nonlinearity to excited-state absorption due to intraband transitions and the decay time to the relaxation of excited carriers to the bottom of the conduction band. Moreover, they observe a 70 ps relaxation time that they ascribe to carrier trapping at surface-localized defects. In our experiment, all these dynamics belong to the instantaneous $\beta_{t=0}$ and $\gamma_{t=0}$ coefficients. For the same sample of Fig. 2, we measure a value of $\gamma_{t=0}=1.0 \times 10^{-16} \mathrm{~m}^{2} / \mathrm{W}$ for the instantaneous nonlinear refraction, which is indeed consistent with the ones quoted in the cited works on samples with comparable Ge-NC density $\left[|\gamma|=3 \times 10^{-17} \mathrm{~m}^{2} / \mathrm{W}\right.$ (Ref. 6) and $\gamma=3.5 \times 10^{-16} \mathrm{~m}^{2} / \mathrm{W}$ (Ref. 7)]. In addition, we evaluate $\beta_{t=0}=4 \times 10^{-10} \mathrm{~m} / \mathrm{W}$, while the cumulative coefficient $\beta_{t=\infty}$ is in the range of $0.6-1.5 \times 10^{-9} \mathrm{~m} / \mathrm{W}$ (Fig. 3). In Ref. $7, \beta=3.5 \times 10^{-9} \mathrm{~m} / \mathrm{W}$ is obtained, a value closer to our $\beta_{t=\infty}$ than to our $\beta_{t=0}$. This fact can be understood considering that their $\beta$ value has been measured with a pulse repetition rate as high as ours and so it could suffer from cumulative contributions as well. In Fig. 3, one can see that both $\gamma_{t=0}$ and $\beta_{t=0}$ have no dependence on the laser intensity thus being pure third-order nonlinearities. This result is in agreement with Refs. 7 and 9. Moreover, the independence of the nonlinearity on the laser intensity was observed with pulse enlicense or copyright; see http://apl.aip.org/about/rights_and_permissions 


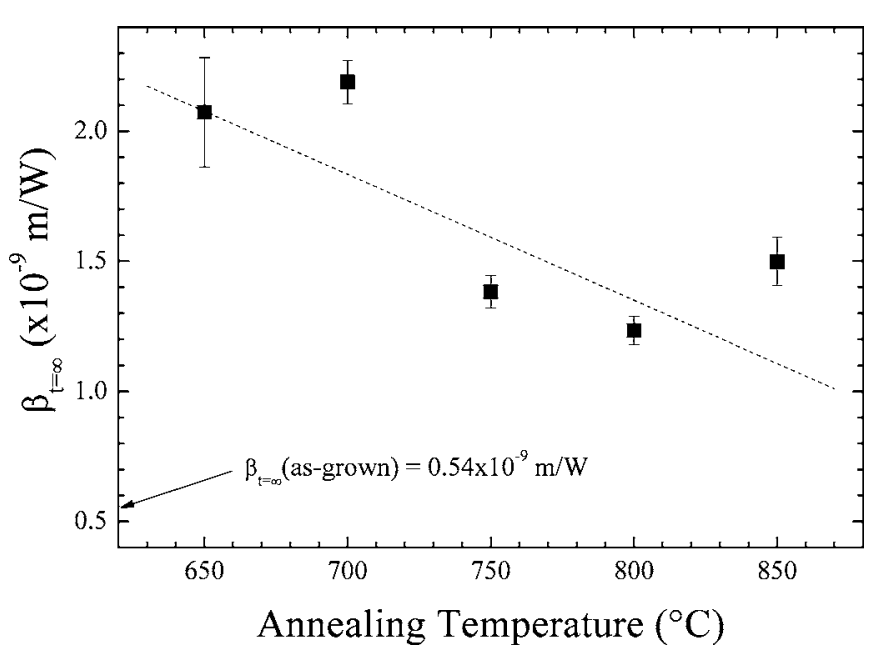

FIG. 4. Cumulative nonlinear absorption coefficient $\beta_{t=\infty}$ as a function of the annealing temperature of the samples. The $\beta_{t=\infty}$ value for an as-grown sample is also indicated.

ergy up to three orders of magnitude higher than ours, ${ }^{9}$ thus reinforcing this picture. Regarding cumulative coefficient $\beta_{t=\infty}$, instead we measure a linear rise with laser intensity up to $8 \times 10^{13} \mathrm{~W} / \mathrm{m}^{2}$, suggesting that three-photon absorption can also have a role in the transitions associated with this coefficient.

According to literature and our results, the NLO observed at $800 \mathrm{~nm}$ in $\mathrm{Ge}-\mathrm{NC}$ can be sketched as follows: (1) linear absorption generates excited carriers in conduction band. Carriers relax to the bottom of the band via phonon scattering in about 1 ps. (2) The excited-state absorption due to intraband transitions produces the instantaneous nonlinearity $\left(\gamma_{t=0}\right.$ and $\left.\beta_{t=0}\right)$. Carrier trapping by a defect state takes place in about 70 ps. (3) The population density of the defect state accumulates since the recombination lifetime $(300 \mu \mathrm{s})$ is longer than the delay between successive laser pulses (13 ns). One- and two-photon absorption from this excited trap state gives rise to the cumulative nonlinear signal $\beta_{t=\infty}$, according to the linear intensity dependence shown in Fig. 3. It is worth noting that, given the fast trapping time, only a single pulse contributes to the population lasting on the conduction band. On the contrary, the population of the trap state is cumulated by tens of thousands of pulses. This difference in population density could explain why we observe a sizable two-photon absorption from the trap state $\left(\beta_{t=\infty}\right)$ and not from the conduction band $\left(\beta_{t=0}\right)$.

The very long lifetime of the trap state is consistent with similar values found for the surface-localized defects in $\mathrm{Si}$ NCs. ${ }^{13}$ Furthermore, charge trapping by defect states at the surface of $\mathrm{Ge}-\mathrm{NC}$ has been experimentally evidenced by charge retention studies on NC-based memory devices ${ }^{14}$ and also theoretically predicted. ${ }^{15}$ In our results, the hypothesis that trap states are localized on the NC surface is supported by the role of the surface-to-volume ratio on the nonlinearity of the Ge-NC. Indeed, the value of $\beta_{t=\infty}$ shows a tendency to decrease on samples annealed at higher temperatures, that is to say, on Ge crystallites with a larger size (Fig. 4). Moreover, since all samples subjected to the annealing procedure show $\beta_{t=\infty}$ larger than that of the as-grown sample, we can state with confidence that the enhancement of the cumulative nonlinearity is induced by Ge clustering.

In conclusion, we have studied the nonlinearity of Ge$\mathrm{NCs}$ embedded in a silica matrix by using a dedicated $Z$-scan setup. We clearly distinguished two nonlinear processes. A fast one confirms preceding measurements of other authors and can be tentatively ascribed to excited-carrier absorption in conduction band. A second process involves carriers trapped at the surface of the Ge-NC. We measured the lifetime of this surface state to be $300 \mu$ s.

The authors thank F. Fernández-Alonso for his critical reading of the manuscript. This work has been partially supported by the EU project SEMINANO under Contract No. NMP4 CT2004 505285 and by TUBITAK through Grant No. TBAG-U/85. A.G. wishes to acknowledge a FIRB-MIUR 2001 Fellowship.

${ }^{1}$ W. K. Choi, W. K. Chim, C. L. Heng, L. W. Teo, Vincent Ho, V. Ng, D. A. Antoniadis, and E. A. Fitzgerald, Appl. Phys. Lett. 80, 2014 (2002); M. Yamamoto, T. Koshikawa, T. Yasue, H. Harima, and K. Kajiyama, Thin Solid Films 369, 100 (2000); J. Xu, Z. H. He, K. Chen, X. Huang, and D. Feng, J. Phys.: Condens. Matter 11, 1631 (1999).

${ }^{2}$ G. P. Banfi, V. Degiorgio, and D. Ricard, Adv. Phys. 47, 447 (1998).

${ }^{3}$ Y. Maeda, N. Tsukamoto, Y. Yazawa, Y. Kanemitsu, and Y. Masumoto, Appl. Phys. Lett. 59, 3168 (1991).

${ }^{4}$ A. G. Cullis, L. T. Canham, and P. D. J. Calcott, J. Appl. Phys. 82, 909 (1997).

${ }^{5}$ J. P. Wilcoxon, P. P. Provencio, and G. A. Samara, Phys. Rev. B 64, 035417 (2001).

${ }^{6}$ A. Dowd, R. G. Elliman, M. Samoc, and B. Luther-Davies, Appl. Phys. Lett. 74, 239 (1999).

${ }^{7}$ H. P. Li, C. H. Kam, Y. L. Lam, Y. X. Jie, W. Ji, A. T. S. Wee, and C. H. A. Wan, Appl. Phys. B: Lasers Opt. 72, 611 (2001).

${ }^{8}$ Y. X. Jie, Y. N. Xiong, A. T. S. Wee, C. H. A. Huan, and W. Ji, Appl. Phys. Lett. 77, 3926 (2000).

${ }^{9}$ A. Dowd, M. Samoc, B. Luther-Davies, and R. G. Elliman, Nucl. Instrum. Methods Phys. Res. B 148, 964 (1999).

${ }^{10}$ Q. Wan, T. H. Wang, and C. L. Lin, Nanotechnology 14, L15 (2003).

${ }^{11}$ M. Sheik-Bahae, A. A. Said, T. H. Wei, D. J. Hagan, and E. W. Van Stryland, IEEE J. Quantum Electron. QE-26, 760 (1990).

${ }^{12}$ A. Gnoli, L. Razzari, and M. Righini, Opt. Express 13, 7976 (2005).

${ }^{13}$ A. Y. Kobitski, K. S. Zhuravlev, H. P. Wagner, and D. R. T. Zahn, Phys. Rev. B 63, 115423 (2001).

${ }^{14}$ E. W. H. Kan, W. K. Choi, W. K. Chim, E. A. Fitzgerald, and D. A. Antoniadis, J. Appl. Phys. 95, 3148 (2004).

${ }^{15}$ Y. M. Niquet, G. Allan, C. Delerue, and M. Lannoo, Appl. Phys. Lett. 77, 1182 (2000). 